University of Warwick institutional repository: http://go.warwick.ac.uk/wrap This paper is made available online in accordance with publisher policies. Please scroll down to view the document itself. Please refer to the repository record for this item and our policy information available from the repository home page for further information.

To see the final version of this paper please visit the publisher's website. Access to the published version may require a subscription.

Author(s): George Rowlands

Article Title: Measurement of penetration depths in superconducting films

Year of publication: 1995

Link to published version: http://dx.doi.org/10.1063/1.113755

Publisher statement: none 


\title{
Measurement of penetration depths in superconducting films
}

\author{
George Rowlands ${ }^{a)}$ \\ Department of Physics, University of Warwick, Coventry CV4 7AL, United Kingdom
}

(Received 6 December 1994; accepted for publication 22 March 1995)

A closed expression is obtained for the mutual inductance of any arrangement of stacked circular coils between which a thin film of superconducting material is inserted. (C) 1995 American Institute of Physics.

Fiory et al. (FHMH) have introduced a method of measuring the penetration depth in superconductors $\lambda$, by relating it to the measurement of the change in mutual inductance caused by the interposition of a superconductor film in a certain coil arrangement. Unfortunately in their treatment, it is necessary to solve an integral equation, involving $\lambda$ as a parameter, before calculating the mutual inductance which is then compared to the experimental measured value. This distracts considerably from what otherwise is a very useful method. Pippard ${ }^{2}$ has recently made this same point and presented an alternate but approximate treatment which gives a more direct relationship between $\lambda$ and the mutual inductance. Here we show that the integral equation of FHMH can be solved exactly, in which case the expression for the mutual inductance reduces to quadrature with $\lambda$ appearing in the integrand.

The basic equation for the sheet current density $\mathbf{K}(\mathbf{r})$ in the film is Eq. (2) of Fiory et al. which, for a cylindrical symmetric arrangement of coils, can be written in the form

$$
\phi(\mathbf{r})=\lambda_{1} A(\mathbf{r})+\lambda_{2} \int_{0}^{\infty} r^{\prime} d r^{\prime} \int_{0}^{2 \pi} \frac{\phi\left(\mathbf{r}^{\prime}\right) \cos \theta d \theta}{\left|\mathbf{r}-\mathbf{r}^{\prime}\right|},
$$

where $\quad \mathbf{K}(\mathbf{r})=\phi(\mathbf{r}) \hat{\theta}, \quad \mathbf{A}_{e}=A(\mathbf{r}) \hat{\theta}, \quad \lambda_{2}=\lambda_{1} / c, \quad$ and $\quad \lambda_{1}$ $=-i \omega / Z c$, where $Z$ is the complex impedance of the film and is given by $Z=R-\omega^{2} \lambda^{2} / d c^{2}$ with $R$ the resistance of the sample. Here, $\omega$ is the frequency of measurement, $d$ the sample thickness, and $\mathbf{A}_{e}$ the magnetic vector potential due to the coils. If we consider the solution of (1) in the plane of the sample, then $\phi$ and $A$ are functions of the radial distance $r$ only. This equation is basically the London equation relating the current to the vector potential. The latter has two contributions, one from the applied field and the other due to the current in the film. Using the identity ${ }^{3}$

$$
\frac{1}{\left|\mathbf{r}-\mathbf{r}^{\prime}\right|}=\sum_{m=-\infty}^{+\infty} \int_{0}^{\infty} d k e^{i m \theta} J_{m}(k r) J_{m}\left(k r^{\prime}\right),
$$

and introducing the Bessel transform

$$
\tilde{\phi}(l)=\int_{0}^{\infty} \phi\left(r^{\prime}\right) J_{1}\left(l r^{\prime}\right) r^{\prime} d r^{\prime},
$$

allows one to reduce Eq. (1) to

$$
\tilde{\phi}(l)=\lambda_{1} \tilde{A}(l)+2 \pi \lambda_{2} \tilde{\phi}(l) / l,
$$

where we have used the result ${ }^{3}$

\footnotetext{
${ }^{a)}$ Electronic mail: physics@uk.ac.warwick.spec
}

$$
\int_{0}^{\infty} r J_{1}(l r) J_{1}(k r) d r=(1 / l) \delta(l-k) .
$$

Here $\delta(l)$ denotes the Dirac delta function. Thus we have obtained an explicit solution to the Fiory et al. integral equation of the form

$$
\phi(r)=\lambda_{1} \int_{0}^{\infty} A\left(r^{\prime}\right) r^{\prime} H\left(r, r^{\prime}\right) d r^{\prime}
$$

where

$$
H\left(r, r^{\prime}\right)=\int_{0}^{\infty} \frac{l^{2} J_{1}(l r) J_{1}\left(l r^{\prime}\right) d l}{l-2 \pi \lambda_{2}}
$$

Following Fiory et al. we define the inductance $M$ such that

$$
M=M_{0} \int_{0}^{\infty} \phi(r) A(r) r d r
$$

and use the above explicit solution to give

$$
M=\lambda_{1} M_{0} \int_{0}^{\infty} \frac{d l l^{2} \tilde{A}^{2}(l)}{\left(l-2 \pi \lambda_{2}\right)} .
$$

This expresses $M$ directly in terms of the magnetic vector potential due to the coils and the $\lambda$ 's which are simply related to the penetration depth. Thus the evaluation of $M$ has been reduced to quadrature. The Pippard approximation is equivalent to expanding the denominator in powers of $2 \pi \lambda_{2} / l$ and retaining just two terms.

I would like to thank Eduardo Palenque who drew my attention to the FHMH method and who has used the present analysis in the design of apparatus to measure $\lambda$.

\footnotetext{
${ }^{1}$ A. T. Fiory, A. F. Hebard, P. M. Mankiewich, and R. E. Haward, Appl. Phys. Lett. 52, 2165 (1988).

${ }^{2}$ A. B. Pippard, Supercond. Sci. Technol. 7, 696 (1994).

${ }^{3}$ J. D. Jackson, Classical Electrodynamics, 2nd ed. (Wiley, New York, 1975), p. 131.
} 